\title{
Liver Suction-Mediated Transfection in Mice Using a Pressure- Controlled Computer System
}

\author{
Kazunori Shimizu, ${ }^{* a, b, \dagger}$ Guangyuan Zhang, ${ }^{c}$ Shigeru Kawakami, ${ }^{d}$ Yota Taniguchi, ${ }^{c}$ \\ Kouji Hayashi, ${ }^{c}$ Mitsuru Hashida, ${ }^{a, c, e}$ and Satoshi Konishi*,a,b,f \\ ${ }^{a}$ Institute for Innovative NanoBio Drug Discovery and Development, Graduate School of Pharmaceutical Sciences, \\ Kyoto University; ${ }^{c}$ Department of Drug Delivery Research, Graduate School of Pharmaceutical Sciences, Kyoto \\ University; 46-29 Yoshida-shimoadachi-cho, Sakyo-ku, Kyoto 606-8501, Japan: ${ }^{b}$ Ritsumeikan-Global Innovation \\ Research Organization, Ritsumeikan University; ${ }^{f}$ Department of Mechanical Engineering, Ritsumeikan University; \\ 1-1-1 Nojihigashi, Kusatsu, Shiga 525-0058, Japan: ' Graduate School of Biomedical Sciences, Nagasaki University; \\ 1-14 Bunkyo-cho, Nagasaki 852-8521, Japan: and ${ }^{e}$ Institute for Integrated Cell-Material Sciences, Kyoto University; \\ Yoshida-ushinomiya-cho, Sakyo-ku, Kyoto 606-8302, Japan.
}

Received October 6, 2013; accepted December 27, 2013; advance publication released online January 15, 2014

We previously developed an in vivo tissue suction-mediated transfection method (denoted as the tissue suction method) for naked nucleic acids, such as plasmid DNA (pDNA) and small interfering RNA (siRNA), in mice. However, it remains unclear whether the suction pressure conditions affect the results of this method. Therefore, in the present study, we assembled a computer system to control the suction pressure and investigate the effects of the suction pressure conditions on the efficiency of the liver suction transfection of naked pDNA that encodes luciferase in mice. Using the developed system, we examined the effects of the minimum magnitude of the suction pressure, suction pressure waveform, and suction times of the luciferase expression level in mice livers. We determined that the liver suction method at $-5 \mathrm{kPa}$ was not only effective but also caused the lowest hepatic toxicity in mice. Additionally, the results indicated that the suction pressure waveform affects the luciferase expression levels, and a single period of suction on the targeted portion of the liver is sufficient for transfection. Thus, the developed system is useful for performing the tissue suction method with high accuracy and safety.

Key words gene delivery; mechanical stimulus; computer system; naked DNA; tissue suction

It is important to develop in vivo transfection methods that can be used for biological research and therapies that use nucleic acids. In vivo transfection methods using recombinant viral vectors or nonviral drug delivery system (DDS) carriers, such as cationic polymers and liposomes, have been investigated widely ${ }^{1-4)}$; however, the methods that deliver naked nucleic acids using physical stimuli are considered the most straightforward because of low toxicities associated with the transfection agents, convenient preparation, ease of handling, and so on. ${ }^{5-7)}$

We have reported a tissue pressure-mediated transfection method (denoted as the tissue pressure method) in which a direct pressure to the kidneys, spleen, and liver can induce efficient gene transfection using naked nucleic acids into each organ. ${ }^{8-10)}$ In this method, the target organs of the mice are pressed directly after intravenous injection (i.v.) of the naked nucleic acids. Previous studies demonstrated that the degree of pressure could be used to control the expression levels of the transfected plasmid DNA (pDNA). ${ }^{9-11)}$ Regarding kidneys in mice, renal dysfunction ${ }^{8)}$ and secretion of proinflammatory cytokines ${ }^{9)}$ were not observed under the experimental conditions of the tissue pressure method. Recently, Oba et al. transfected the miR-200 family of microRNA to mice kidneys via the tissue pressure method and reported that that transfection ameliorated renal tubulointerstitial fibrosis in the mice. ${ }^{12)}$ Pons et al. used the tissue pressure method to transfect pDNA

\footnotetext{
The authors declare no conflict of interest.

${ }^{\dagger}$ Present address: Division of Chemical Engineering, Graduate School of Engineering Science, Osaka University; 1-3 Machikaneyama-cho, Toyonaka, Osaka 560-8531, Japan.
}

encoding heat shock protein 70 (HSP70) to rat kidneys and reported that the immune reactivity to HSP70 expressed in the kidney is cause of salt-sensitive hypertension. ${ }^{13)}$ Therefore, the tissue pressure method is beneficial for biological research using small laboratory animals, such as mice and rats. However, the method must be improved for application in humans in the future.

Considering the potential clinical uses, we have developed an in vivo tissue suction-mediated transfection method (denoted as the tissue suction method). ${ }^{14)}$ Its procedure is different from the tissue pressure method: the target site of the tissue is supplied with a suction pressure from tissue suction microdevices after the i.v. administration of naked nucleic acids in mice. Because the microdevices can be mounted at the head of an endoscope, ${ }^{15)}$ this method can be performed in a minimally invasive manner by exploiting endoscopic surgery. Transfection was achieved by the suction of the mouse kidney, liver, spleen, and heart; severe toxicity and tissue damage were not observed in the mouse liver suctioned with the appropriate methodology. ${ }^{14)}$

In the previous study, we performed the tissue suction method with uncontrolled suction pressure, ${ }^{14)}$ that is, we supplied a negative pressure with the tissue suction device manually by using a syringe until the inner space of the device was filled with the deformed tissue. It remains unclear whether the suction pressure conditions affect the results of the tissue suction method. To perform the tissue suction method safely and accurately at research facilities and medical institutions, the suction method must be performed with a controlled suction pressure. Therefore, in the present study, we assembled a computer system to control the suction pressure and investigate 
the effects of the suction pressure conditions on the efficiency of the liver suction transfection of naked pDNA in mice.

\section{MATERIALS AND METHODS}

Fabrication of Tissue Suction Devices The tissue suction devices were manufactured via a polydimethylsiloxane (PDMS) replica molding process. The molds were fabricated with a 3D printing system (Objet Geometries Ltd., Rehovot, Israel) and coated with Parylene C (Specialty Coating Systems, Inc., Indianapolis, IN, U.S.A.) as described in a previous report. ${ }^{16)}$ The precured PDMS $(20: 1)$ was cured at $75^{\circ} \mathrm{C}$ for $12 \mathrm{~h}$ in the molds and the cured PDMS was peeled off and cut into individual devices. Then, an individual device was punched out using a disposable biopsy punch (Kai Industries Co., Ltd., Gifu, Japan), and a silicone tube with an outer diameter of $2 \mathrm{~mm}$ was connected to the device. The tube was used to supply the negative pressure. Unless mentioned otherwise, the device sizes had an inner diameter of $3 \mathrm{~mm}$, an outer diameter of $5 \mathrm{~mm}$, and a height of $3 \mathrm{~mm}$ (Fig. 1A).

Suction Pressure Control Computer System Figure 1B shows the block diagram of the suction pressure control system. The negative pressure was generated by a vacuum pump. An electropneumatic regulator (ITV0090; SMC Corp., Tokyo, Japan) was controlled by a PC with specially designed LabVIEW software (National Instrument, Austin, TX, U.S.A.). The LabVIEW software was developed to record the actual suction pressure monitored by the pressure sensor (Sensez Corp., Tokyo, Japan). The suction pressure waveform was defined as a trapezoidal pulse, as shown in Fig. 1C.

pDNA and Mice The pDNA was amplified, isolated, and purified as described in a previous study. ${ }^{17)}$ pDNA encoding complementary luciferase DNA that was driven by a cytomegalovirus (CMV) immediate-early promoter was employed (pCMV-Luc). ${ }^{17)}$ Five-week-old ICR mice (female) were purchased from Japan SLC Inc. (Shizuoka, Japan). All animal experiments were carried out in accordance with the Guide for the Care and Use of Laboratory Animals, as adopted and promulgated by the U.S. National Institute of Health (Bethesda, MD, U.S.A.). The study protocol permission numbers 2012-50 were approved by the Animal Research Committee, Kyoto University, Japan.

In Vivo Transfection by Tissue Suction The mice were anesthetized with isoflurane, ensuring minimal exposure of the liver. Thereafter, $100 \mu \mathrm{g}$ of pCMV-Luc dissolved in $200 \mu \mathrm{L}$ of saline was injected intravenously into the mice. The targeted portion of the left liver lobe was suctioned using the device with a negative pressure, which was controlled by the suction pressure control system. Six hours after tissue suction, the left lobe was harvested and the gene expression levels were determined by the luciferase assay, as previously described. ${ }^{10,14,17)}$

Transaminase Activity in Serum Serum was collected from the mice after 6,24 , and $48 \mathrm{~h}$ of the liver suction. The alanine transaminase (ALT) and aspartate transaminase (AST) activities in the serum were measured, as described previously. ${ }^{18)}$

Statistical Analysis Prism 5 software (Graphpad Software, La Jolla, CA, U.S.A.) was used for statistical analysis.

\section{RESULTS AND DISCUSSION}

Development of Suction Pressure Control Computer System To perform the tissue suction method under controlled suction pressure conditions, we set up a suction pressure control computer system (Fig. 1B). The minimum magnitude of the suction pressure ( $\mathrm{a}$ in Fig. 1C), pressure supply time (b), pressure hold time (c), and pressure release time (d) were input to an original LabVIEW program to define the suction pressure waveform. Because the controlled suction pressure is generated as defined in the program by turning on a foot switch, the operators can use both hands to perform the tissue suction method. The actual suction pressure waveform supplied to the tissue is monitored by a pressure sensor.

Figure 2 shows the results of the operation conditions. First, we investigated the controllability of the minimum magnitude of the suction pressure, which was set to $-1,-3,-5,-15$, and $-30 \mathrm{kPa}$. The pressure supply time, pressure hold time, and pressure release time were set to 1,3 , and $1 \mathrm{~s}$, respectively (1-3-1). As shown in Fig. 2A, the minimum magnitude of the actual suction pressure, which was monitored by the sen-
A

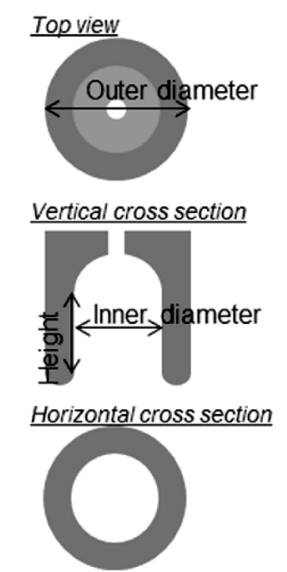

B

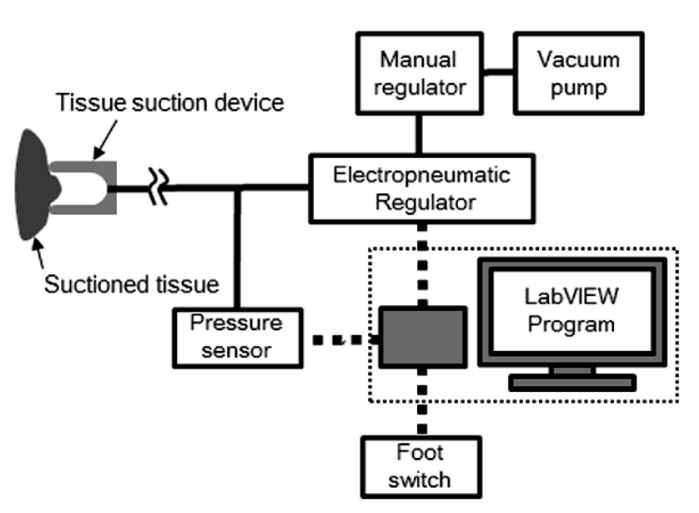

C

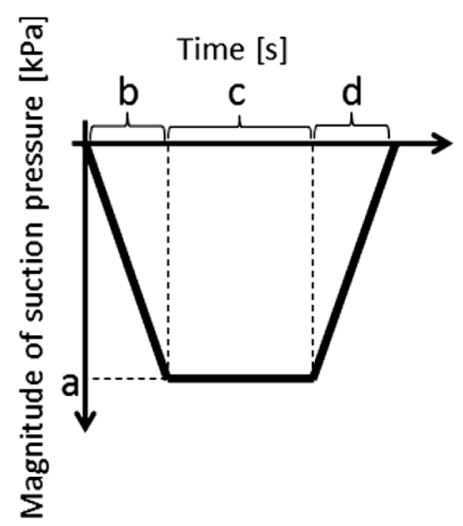

Fig. 1. The Tissue Suction Method Using the Suction Pressure Control System

(A) Design of the tissue suction device. The device sizes had an inner diameter of $3 \mathrm{~mm}$, an outer diameter of $5 \mathrm{~mm}$, and a height of $3 \mathrm{~mm}$. (B) Configuration of the suction pressure control computer system. (C) Suction pressure waveform: a, minimum magnitude of the suction pressure; b, pressure supply time; c, pressure hold time; d, pressure release time. 
A

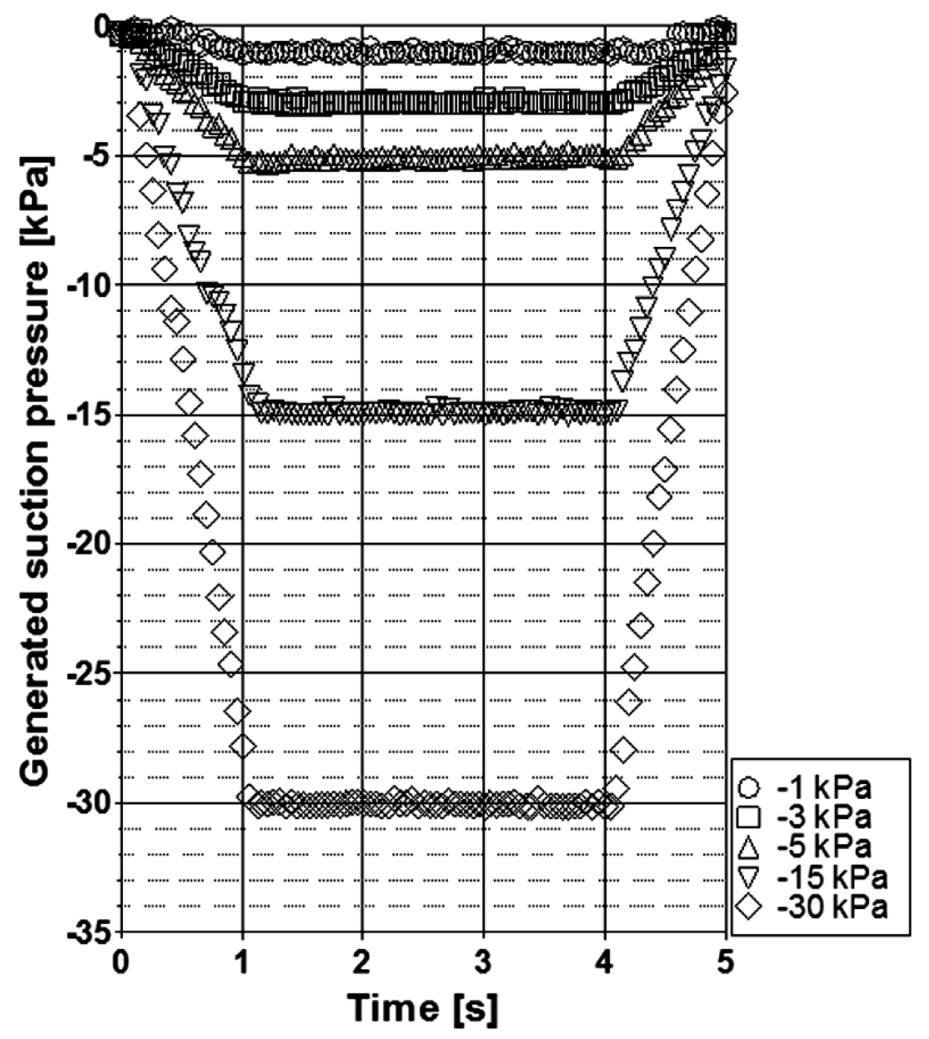

$\mathrm{B}$ (i)

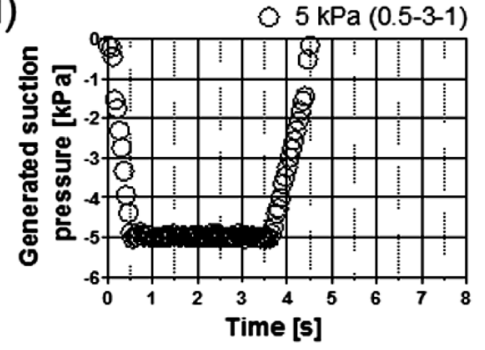

(ii)

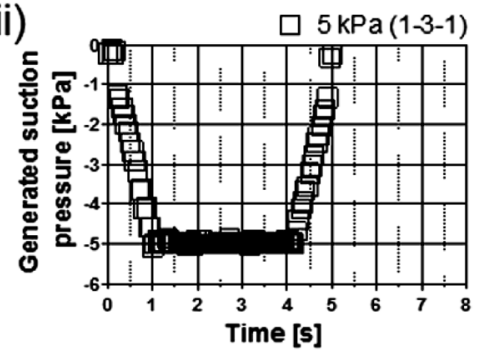

(iii)

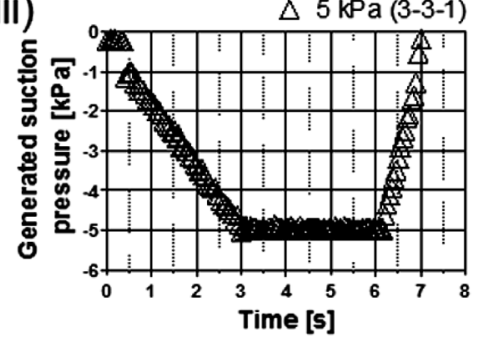

Fig. 2. Operation Conditions of the Developed System

(A) Minimum magnitudes of the suction pressure of $-1,-3,-5,-15$, and $-30 \mathrm{kPa}$. (B) Pressure supply times of (i) $0.5 \mathrm{~s}$, (ii) $1 \mathrm{~s}$, and (iii) $3 \mathrm{~s}$.

sor, was similar to the input minimum magnitude of suction pressure. Secondly, we investigated the controllability of the pressure supply time, which was set to $0.5,1$, and $3 \mathrm{~s}$ (b-3-1, where $b=0.5,1,3 \mathrm{~s})$. For this case, the minimum magnitude of the suction pressure was set to $-5 \mathrm{kPa}$. As shown in Fig. 2B, the actual pressure supply time and suction pressure waveform, which were monitored by the sensor, were similar to the programmed pressure supply time and the waveform that was input to the program, respectively. Thus, the suction pressure could be controlled by using the developed system.

Effects of Suction Pressure Magnitude on Luciferase Level in Mice Livers We elucidated whether the magnitude of the suction pressure affects the expression level of transfected naked pDNA when using the developed system. The minimum magnitudes of the suction pressure were set to -1 , $-3,-5,-15,-30$, and $-40 \mathrm{kPa}$. The pressure supply time, pressure hold time, and pressure release times were set to 1 , 3 , and $1 \mathrm{~s}$, respectively (1-3-1). As shown in Fig. 3, the luciferase level increased as the minimum magnitude of the suction pressure decreased, reaching a constant level at less than $-5 \mathrm{kPa}$. Thus, the expression levels of transfected pDNA are controlled by the minimum magnitude of the suction pressure. Similarly, we previously reported that the expression levels of pDNA transfected using the tissue pressure method are controlled by the magnitude of the positive pressure; $0.59 \mathrm{~N} / \mathrm{cm}^{2}$ $(5.9 \mathrm{kPa})$ was sufficient to achieve efficient and highly reproducible pDNA transfection using the tissue pressure method for kidneys and spleens in mice. ${ }^{9)}$ Although the absolute values of the positive and negative pressures are similar, it is

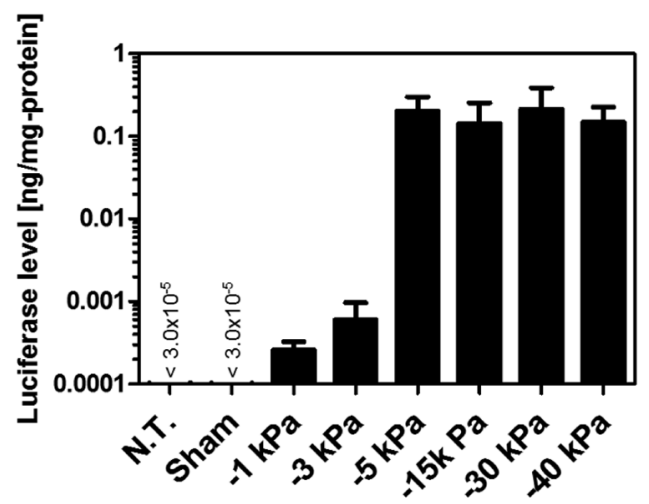

Fig. 3. Effects of the Minimum Magnitude of the Suction Pressure on the Transgene Expression Level

Each value represents mean \pm S.D. $(n=4)$.

still unknown whether the similarity is significant because the studies were performed on different target tissues. This will be clarified in the future.

Next, we examined the effects of liver suction on the hepatic toxicity for different minimum magnitudes of suction pressure. Considering the results in Fig. 3, the following effective magnitudes of the suction pressure were employed in this experiment: $-5,-15,-30$, and $-40 \mathrm{kPa}$. Both ALT and AST activities in the serum were measured $6 \mathrm{~h}$ after the liver suction. The ALT activities at $-5,-15$, and $-30 \mathrm{kPa}$ were not significantly different from that at $0 \mathrm{kPa}$ (Sham), whereas the activity at $-40 \mathrm{kPa}$ was significantly higher than that at 
0 and $-5 \mathrm{kPa}$ (Fig. 4A). The AST activities increased with a decrease in the minimum magnitude of the suction pressure and the activity at $-5 \mathrm{kPa}$ was significantly higher than that at $0 \mathrm{kPa}$ (Fig. 4B). However, the AST activities returned to normal levels within $24 \mathrm{~h}(-5 \mathrm{kPa}, 56.7 \pm 9.6 \mathrm{U} / \mathrm{L}$; Sham, $52.7 \pm$ 7.6U/L). Considering these results, the liver suction method at $-5 \mathrm{kPa}$ was the most effective and had the lowest hepatic toxicity in mice.

In the previous study, we reported that both ALT and AST activities $6 \mathrm{~h}$ after liver suction with an uncontrolled suction pressure were significantly higher than the activities in mice with $0 \mathrm{kPa}$, and the activities returned to normal levels within $48 \mathrm{~h}^{14)}$ In contrast, in the present study, when the liver suction method in mice was performed at $-5 \mathrm{kPa}$, an increase in ALT activity was not observed and the increased AST activity quickly returned to normal levels within $24 \mathrm{~h}$. Therefore, the toxicity of the liver suction method was reduced by using the suction pressure control system.

Effects of Suction Pressure Waveform on Luciferase Level in Mice Livers Next, we investigated the effects of the suction pressure waveform on the expression level of pDNA transfected by liver suction. The minimum magnitude of the suction pressure was set to $-5 \mathrm{kPa}$. The pressure supply time and the pressure hold time were both varied. High luciferase levels were measured when the pressure supply time was set to $0.5 \mathrm{~s}$ at all the pressure hold times, and statistically significant differences were observed between 0.5 and $3 \mathrm{~s}$ for
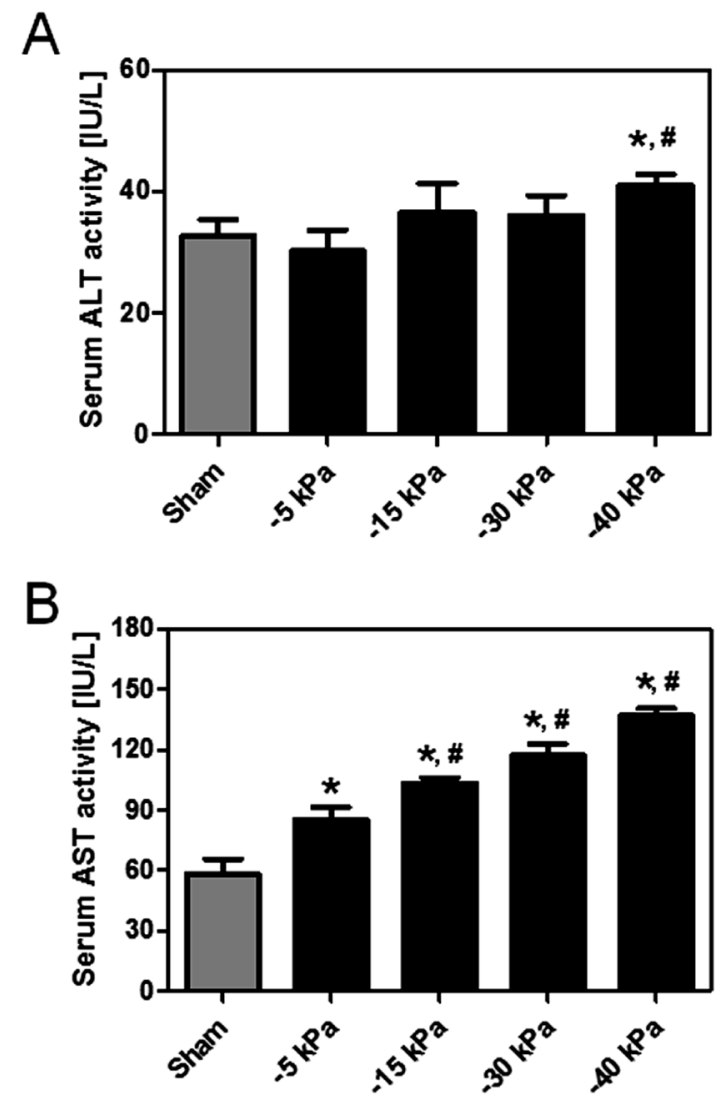

Fig. 4. Effects of the Minimum Magnitude of the Suction Pressure on Hepatic Toxicity

(A) ALT and (B) AST activities in serum $6 \mathrm{~h}$ after the liver suction. Each value represents mean \pm S.D. $(n=4)$. One-way ANOVA, followed by Bonferroni's test was used to compare among groups. ${ }^{*} p<0.05$ versus Sham operation. ${ }^{*} p<0.05$ versus $-5 \mathrm{kPa}$. both the 1- and 2-s pressure hold times. The luciferase levels at 1,2 , and $3 \mathrm{~s}$ were significantly higher than that at $0 \mathrm{~s}$ when the pressure supply time was $0.5 \mathrm{~s}$ (Fig. 5), and a constant luciferase level was maintained when the hold time was $30 \mathrm{~s}$ (data not shown).

A shorter pressure supply time increased the transgene expression in the suctioned mice livers (Fig. 5). In a previous study, we reported that the pDNA permeability of the plasma membranes in the pressed tissues transiently increased. ${ }^{10}$ ) This phenomenon may be related to the increased transgene expression owing to the shorter pressure supply time observed in the present study. In the field of neural injury, the responses of cells and tissues to mechanical loading have been investigated ${ }^{19)}$ and in vivo ${ }^{20)}$ and in vitro ${ }^{21)}$ studies reported that the mechanical deformations of cells and tissues form transient survivable pores on the plasma membrane, resulting in the transient increase of permeability. More recently, in vitro experimental models of neural injuries showed an increase in the permeability with respect to the strain rate; a higher strain rate effectively alters the membrane permeability. ${ }^{22,23)}$ Thus, similar tendencies may occur for these different phenomena. However, further experiments should be performed to explain the phenomenon of cell membrane permeability in the field of neural injury and studies regarding the tissue suction method.

There is possibility that a pressure supply time of less than $0.5 \mathrm{~s}$ may increase the luciferase levels (Fig. 5). However, the tissue suction method could not be performed with a pressure supply time of less than $0.5 \mathrm{~s}$ because our pneumatic system could not control times lower than $0.5 \mathrm{~s}$. Thus, the suction pressure control system can be further modified to include a rapid response speed, such as the use of hydraulic pressure systems. Nonetheless, in the present study, we determined that the pressure waveform affects the efficiency of the tissue suction method in mice livers and the most effective suction method was performed with a suction pressure waveform of 0.5-1-1 when the minimum magnitude of the suction pressure was $-5 \mathrm{kPa}$.

Effects of Tissue Suction Times on Luciferase Level in Mice Livers In the previous study, we performed the liver



Fig. 5. Effects of the Suction Pressure Waveform on the Transgene Expression Level

Each value represents mean \pm S.D. $(n=4)$. Two-way ANOVA, followed by Bonferroni's test was used to compare among groups. $* p<0.05$ versus $3 \mathrm{~s}$ of the pressure supply time for the same pressure hold time. ${ }^{\#} p<0.05$ versus $0 \mathrm{~s}$ of the pressure hold time for the same pressure supply time. 
suction method by subsequently or serially suctioning different parts of the liver and reported that the transgene expression levels increased with an increase in the tissue suction number for both cases. ${ }^{14)}$ In contrast, in the present study, we examined whether the number of tissue suctions on the same part of the liver increases the expression levels of the pDNA. We suctioned the same part of the mice liver 1, 3, 5, or 10 times immediately after i.v. injection of the pDNA solution. Because of the developed suction pressure control system, we were able to perform the tissue suction method on the same part repeatedly with the same conditions. As shown in Fig. 6, the number of liver suctions did not alter the luciferase levels of the liver. This result suggests that a single suction on the targeted portion of the tissue is sufficient for the liver suction method in mice.

Effect of Tissue Deformation on Luciferase Level in Mice Livers The minimum magnitude of the suction pressure affected the transgene expression in the liver suction method (Fig. 3). Similarly, the results of the previous study showed that the maximum magnitude of the positive pressure affected the transgene expression of mice kidneys and spleens in the tissue pressure method. ${ }^{9)}$ Because both the positive and

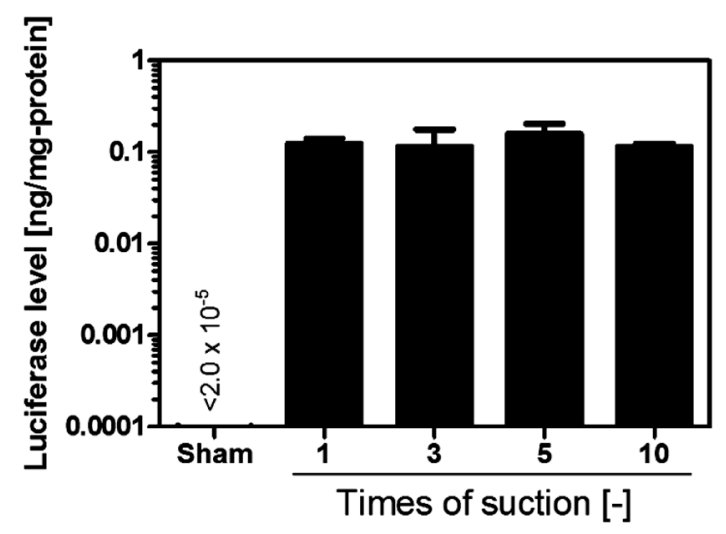

Fig. 6. Effects of the Number of Liver Suctions

The same part of the liver was suctioned serially. Each value represents a mean \pm S.D. $(n=3)$. suction pressures induced transfection in the target tissue, we hypothesized that one of the significant factors of the tissue pressure/suction methods is not the pressure, but the deformation of the tissue. To prove this hypothesis, we measured the volume of the suctioned hepatic tissue in the hole of the suction device for suction pressures of $-1,-3,-5$, and $-15 \mathrm{kPa}$. As shown in Fig. 7, a difference was observed in the suctioned volume at the pressures of -3 and $-5 \mathrm{kPa}$, but did not reach significance. A difference in transgene expression levels also existed between the pressures of -3 and $-5 \mathrm{kPa}$ using the tissue suction method (Fig. 3).

Furthermore, we controlled the tissue deformation using four different tissue suction devices and investigated the effects of those devices on the transgene expression. The devices had different numbers of holes but their total dimensions were equivalent (Fig. 8A). In the liver suction method, even though an equivalent pressure of $-5 \mathrm{kPa}$ was supplied to the device, the highest luciferase level was detected for the device with one large hole and the second highest level was observed

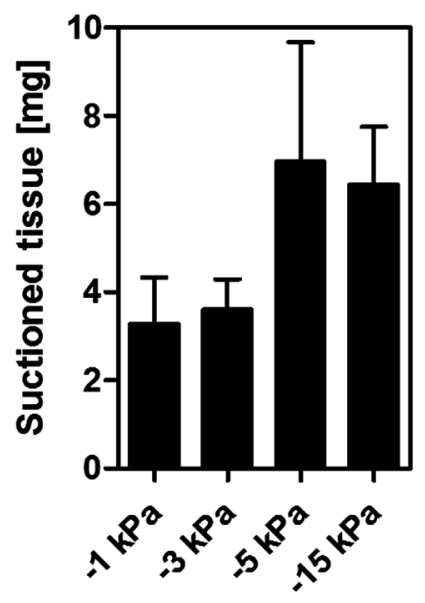

Fig. 7. Volume of the Suctioned Liver Tissue in the Hole of the Suction Device

Volume of the suctioned liver tissue when the mice liver was subject to suction pressures of $-1,-3,-5$, and $-15 \mathrm{kPa}$ using the suction device. Each value represents mean \pm S.D. $(n=3)$.
A

(i)

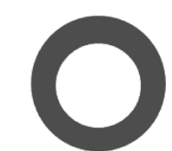

(iii)



(ii)

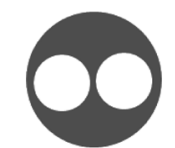

(iv)
B

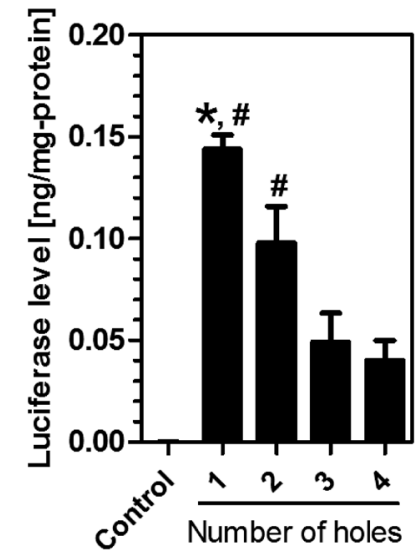

C

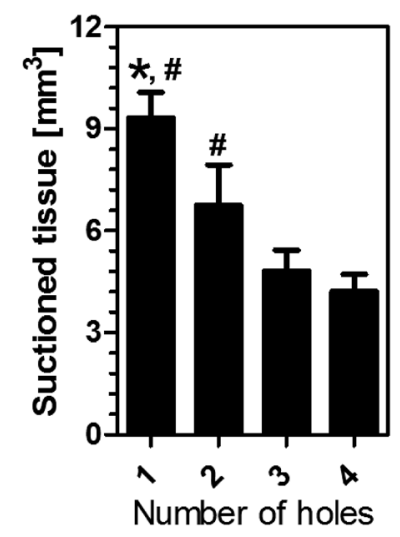

Fig. 8. Relationship between the Degree of Tissue Deformation and the Transgene Expression Level

(A) Horizontal cross section of the four different tissue suction devices with different numbers of holes. The device with one hole had an inner diameter of 3 mm, an outer diameter of $6 \mathrm{~mm}$, and a height of $3 \mathrm{~mm}$. The four devices had the same total dimensions of the holes. (B) Transgene expression level in the suctioned liver after using a different suction device. The minimum magnitude of the suction pressure was $-5 \mathrm{kPa}$ and the pressure waveform was $0.5-1-1$. ${ }^{*} p<0.05$ versus 2,3 , and 4 holes. ${ }^{\#} p<0.05$ versus 3 and 4 holes. (C) Suctioned tissue volumes when the hepatic tissue was suctioned by using devices with different number of holes at the pressure of $-5 \mathrm{kPa} .{ }^{*} p<0.05$ versus 2,3 , and 4 holes. ${ }^{\#} p<0.05$ versus 3 and 4 holes. 
for the device with two holes (Fig. 8B). Similarly, among the volumes of tissue suctioned with the four devices, the volume of the tissue suctioned with the device with one large hole was the largest and that suctioned with the device with two holes was the second largest (Fig. 8C). These results support our hypothesis that the tissue deformation is a key parameter affecting the tissue pressure/suction method. Thus, the process of the tissue suction method has been clarified in part by using the developed suction pressure control system.

Potential Applications of the Liver Suction Method The tissue suction method can be performed in a minimally invasive manner by exploiting endoscopic surgery, because tissue suction devices can be mounted at the head of an endoscope. ${ }^{15)}$ The present study shows that the tissue suction method using a suction pressure control system can be accurately and safely applied for in vivo transfection of naked nucleic acids. These results suggest that the tissue suction method has the potential for use in clinical applications in the future.

One of the features of the tissue suction method is the site-specificity: this method enables a simple and precise sitespecific transfection to the target part of the moving tissues, because the negative pressure easily fixes the position of the tissue relative to the device. ${ }^{14)}$ In addition, the transgene expression levels were increased by suctioning different parts of the tissues. ${ }^{14)}$ Therefore, we can control the transgene expression level in the suctioned tissue while avoiding transfection into the non-target parts of the tissue.

To date, treatment with the tissue suction method has not been investigated. Transfection of naked plasmid DNA encoding hepatocyte growth factor (HGF), which is a secretory protein, has been reported to prevent endotoxin-induced lethal fulminant hepatic failure, leading to dramatically enhanced survival in mice. ${ }^{24}$ Therefore, the use of nucleic acid-based drugs that alter the expression of secretory factors in combination with the tissue suction method might be a good treatment strategy. In the future, we will investigate the appropriate combination of diseases and nucleic acid-based drugs for which the treatment with the tissue suction method could work efficiently and effectively.

\section{CONCLUSION}

We developed a pressure-controlled computer system for the suction-mediated transfection of livers in mice. Our experimental results indicate that the minimum magnitude of the suction pressure and the suction pressure waveform both affected the expression levels of the pDNA transfected via the tissue suction method applied to livers in mice. Our developed system can perform the tissue suction-mediated transfection method with high accuracy and safety at research facilities and medical institutions.

Acknowledgments The authors would like to thank to Dai Maekawa and Shingo Katano for their technical assistance. This work was supported in part by the Health and Labour Sciences Research Grants for Research on Medical Device Development from the Ministry of Health, Labour and Welfare of Japan and by a Grant from the Ritsumeikan Global Innovation Research Organization (R-GIRO).

\section{REFERENCES}

1) Takemoto H, Ishii A, Miyata $K$, Nakanishi M, Oba M, Ishii T, Yamasaki Y, Nishiyama N, Kataoka K. Polyion complex stability and gene silencing efficiency with a siRNA-grafted polymer delivery system. Biomaterials, 31, 8097-8105 (2010).

2) Miller DG, Wang PR, Petek LM, Hirata RK, Sands MS, Russell DW. Gene targeting in vivo by adeno-associated virus vectors. Nat. Biotechnol., 24, 1022-1026 (2006).

3) Kwon SM, Nam HY, Nam T, Park K, Lee S, Kim K, Kwon IC, Kim J, Kang D, Park JH, Jeong SY. In vivo time-dependent gene expression of cationic lipid-based emulsion as a stable and biocompatible non-viral gene carrier. J. Control. Release, 128, 89-97 (2008).

4) Kim A, Lee EH, Choi SH, Kim CK. In vitro and in vivo transfection efficiency of a novel ultradeforinable cationic liposome. Biomaterials, 25, 305-313 (2004)

5) Kamimura K, Liu DX. Physical approaches for nucleic acid delivery to liver. AAPS J., 10, 589-595 (2008).

6) Gao X, Kim KS, Liu DX. Nonviral gene delivery: What we know and what is next. AAPS J., 9, E92-E104 (2007).

7) Mehier-Humbert S, Guy RH. Physical methods for gene transfer: Improving the kinetics of gene delivery into cells. Adv. Drug Deliv. Rev., 57, 733-753 (2005).

8) Mukai H, Kawakami S, Hashida M. Renal press-mediated transfection method for plasmid DNA and siRNA to the kidney. Biochem. Biophys. Res. Commun., 372, 383-387 (2008).

9) Mukai H, Kawakami S, Kamiya Y, Ma F, Takahashi H, Satake K, Terao K, Kotera H, Yamashita F, Hashida M. Pressure-mediated transfection of murine spleen and liver. Hum. Gene Ther., 20, 1157-1167 (2009)

10) Mukai H, Kawakami S, Takahashi H, Satake K, Yamashita F, Hashida M. Key physiological phenomena governing transgene expression based on tissue pressure-mediated transfection in mice. Biol. Pharm. Bull., 33, 1627-1632 (2010).

11) Shimizu K, Kawakami S, Hayashi K, Mori Y, Hashida M, Konishi S. Implantable pneumatically actuated microsystem for renal pressure-mediated transfection in mice. J. Control. Release, 159, 85-91 (2012)

12) Oba S, Kumano S, Suzuki E, Nishimatsu H, Takahashi M, Takamori H, Kasuya M, Ogawa Y, Sato K, Kimura K, Homma Y, Hirata Y, Fujita T. miR-200b precursor can ameliorate renal tubulointerstitial fibrosis. PLoS ONE, 5, e13614 (2010).

13) Pons H, Ferrebuz A, Quiroz Y, Romero-Vasquez F, Parra G, Johnson RJ, Rodriguez-Iturbe B. Immune reactivity to heat shock protein 70 expressed in the kidney is cause of salt-sensitive hypertension. Am. J. Physiol. Renal Physiol., 304, F289-F299 (2013).

14) Shimizu K, Kawakami S, Hayashi K, Kinoshita H, Kuwahara K, Nakao K, Hashida M, Konishi S. In vivo site-specific transfection of naked plasmid DNA and siRNAs in mice by using a tissue suction device. PLoS ONE, 7, e41319 (2012).

15) Konishi S, Horie T, Kurumi Y, Tani T. Reliable positioning of micro device for medical diagnosis and operation on pulsating targets by pneumatic suction device. Journal of Japan Society of Computer Aided Surgery, 11, 59-64 (2009).

16) Shimizu K, Kusamori K, Nishikawa M, Mizuno N, Nishikawa T, Masuzawa A, Katano S, Takahashi Y, Takakura Y, Konishi S. Poly( $N$-isopropylacrylamide)-coated microwell arrays for construction and recovery of multicellular spheroids. J. Biosci. Bioeng., 115, 695-699 (2013)

17) Kawakami S, Fumoto S, Nishikawa M, Yamashita F, Hashida M. In vivo gene delivery to the liver using novel galactosylated cationic liposomes. Pharm. Res., 17, 306-313 (2000)

18) Un K, Kawakami S, Suzuki R, Maruyama K, Yamashita F, Hashida M. Enhanced transfection efficiency into macrophages and dendritic cells by a combination method using mannosylated lipoplexes and bubble liposomes with ultrasound exposure. Hum. Gene Ther., 21, 
65-74 (2010).

19) Barbee KA. Mechanical cell injury. Ann. N.Y. Acad. Sci., 1066, 67-84 (2005).

20) Pettus EH, Christman CW, Giebel ML, Povlishock JT. Traumatically induced altered membrane-permeability-Its relationship to traumatically induced reactive axonal change. J. Neurotrauma, 11, 507-522 (1994).

21) Ellis EF, Mckinney JS, Willoughby KA, Liang S, Povlishock JT. A new model for rapid stretch-induced injury of cells in cultureCharacterization of the model using astrocytes. J. Neurotrauma, 12, 325-339 (1995).
22) Geddes DM, Cargill RS 2nd, LaPlaca MC. Mechanical stretch to neurons results in a strain rate and magnitude-dependent increase in plasma membrane permeability. J. Neurotrauma, 20, 1039-1049 (2003).

23) Cullen DK, Vernekar VN, LaPlaca MC. Trauma-induced plasmalemma disruptions in three-dimensional neural cultures are dependent on strain modality and rate. J. Neurotrauma, 28, 2219-2233 (2011).

24) Liu F, Huang L. Noninvasive gene delivery to the liver by mechanical massage. Hepatology, 35, 1314-1319 (2002). 